\section{Gas tamponade combined with laser photocoagulation therapy for congenital optic disc pit maculopathy}

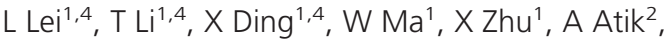
$\mathrm{Y} \mathrm{Hu}{ }^{1}$ and $\mathrm{S}$ Tang ${ }^{1,3}$
${ }^{1}$ State Key Laboratory of Ophthalmology, Zhongshan Ophthalmic Center, Sun Yatsen University, Guangzhou, China

${ }^{2}$ Royal Victorian Eye and Ear Hospital, Melbourne, VIC, Australia

${ }^{3}$ Aier School of Ophthalmology, Central South University, Changsha, China

Correspondence: S Tang, Aier School of Ophthalmology, Central South University, Floor 4, New Century Building, 198\# Furong Middle Road, Changsha 410015,

Hunan, China.

Tel: +86073185179288 8809:

Fax: +86 073182570202

Email: tangshibo@

vip.163.com

${ }^{4}$ These authors contributed equally to this work and are to be considered as first coauthors.

Received: 31 July 2014 Accepted in revised form: 15 September 2014 Published online: 17 October 2014
Abstract

Purpose To evaluate the long-term clinical efficacy and safety of gas tamponade combined with laser photocoagulation for optic disc pit maculopathy.

Methods Seven consecutive patients with unilateral maculopathy associated with optic disc pit and one patient with bilateral optic disc pit maculopathy were given octafluoropropane $\left(\mathrm{C}_{3} \mathrm{~F}_{8}\right)$ tamponade combined with focal laser photocoagulation treatment. Patients were followed up for 21-62 months after treatment. Main outcomes were determined by optical coherence tomography (OCT) and bestcorrected visual acuity (BCVA).

Results Treatment with $\mathrm{C}_{3} \mathrm{~F}_{8}$ tamponade followed by laser photocoagulation in ODP maculopathy patients resulted in resolution of sub-retinal and/or intra-retinal fluid in six out of eight patients. The remaining two patients had significant reduction in fluid, as determined by OCT, and funduscopy, as well as an improvement in anatomical architecture. Visual acuity improved obviously in seven eyes and remained stable in two eyes. Central visual field loss after photocoagulation was not clinically appreciable by visual field examination. No post-operative complications of maculopathy occurred during the followup period.

Conclusions Although repeated treatment was needed in some patients, $\mathrm{C}_{3} \mathrm{~F}_{8}$ tamponade combined with laser photocoagulation is still a simple, effective, minimally invasive, and economic therapy for optic disc pit maculopathy. Eye (2015) 29, 106-114; doi:10.1038/eye.2014.245; published online 17 October 2014
Introduction

Optic disc pit (ODP) is part of a spectrum of congenital cavitary optic disc anomalies which was seen in $<1 / 11000$ patients. ${ }^{1}$ The pit lesions are typically unilateral, oval, and gray-white depressions located in the temporal segment of the optic disc and frequently involve the macula via intra-retinal and sub-retinal fluid collections. ${ }^{2}$ Visual acuity and visual fields are normal unless associated with serous macular detachment.

The mechanisms underlying this disease are unclear, especially in relation to intra-retinal and sub-retinal fluid accumulation. ODP maculopathy (ODPM) was first described by Halbertsma ${ }^{1}$, with macular changes including serous detachment, cystic degeneration, and degenerative pigment retinopathy. Serous macular detachment or schisis develops in $\sim 25-75 \%$ of patients with visual deterioration. ${ }^{3}$ Although spontaneous reattachment has been reported ${ }^{4,5}$ the prognosis of ODPM is poor if left to its natural course. ${ }^{6}$ Several therapeutic approaches have been attempted, but responses have been variable. A relatively high incidence of recurrence has been reported after conventional laser photocoagulation or gas tamponade alone..$^{7-9}$ Vitrectomy with or without a combination of laser treatment and/or gas tamponade has been reported to be an effective treatment and is widely used. ${ }^{10-13}$ However, the potential complications of vitrectomy in ODPM patients (eg, macular hole), ${ }^{11}$ and controversy surrounding appropriate adjunct procedures (eg, induction of posterior vitreous detachment (PVD) or internal limiting membrane (ILM) peeling) means that there is still no standard treatment for ODPM. 
Lincoff $^{9}$ described the association between ODP and schisis-like separation of the macula. He suggested that fluid initially enters the retinal stroma to form a schisis, and then enters the sub-retinal space to eventually cause a serous retinal detachment. However, the precise origin of the sub-retinal fluid remains elusive. Several possible sources have been suggested, including cerebrospinal fluid, liquefied vitreous, ${ }^{3,14}$ or leaky vessels at the base of the pit. ${ }^{2}$ Postel et al ${ }^{15}$ also observed some cases with a subtle tear in the diaphanous tissue overlying the optic pit, suggesting a rhegmatogenous component to the detachment. In either case, the sub-retinal fluid appears to gain access to the macula via the anomalous optic nerve.

The advent of optical coherence tomography (OCT) has allowed a greater understanding of the pathophysiology of ODPM. ${ }^{16}$ By using spectral domain OCT with eye tracking technology, studies have confirmed that fluid from the optic pit can go directly into the retina. ${ }^{16}$ We hypothesize that this macular fluid can be blocked by forming a barrier in the region of the pit and extending into the central macula. This barrier can be achieved by hydrostatic pressure from intravitreal gas tamponade (which can force the absorption of the macular fluid) and laser photocoagulation (which can cause full-thickness scarring of the retina along the optic pit).

Although combined treatment with gas tamponade and laser has previously been reported, 17,18 these studies performed laser first followed by gas tamponade and only had small case series and short-term follow-up. Thus, we report the first long-term clinical study on the safety and efficacy of gas tamponade using octafluoropropane $\left(\mathrm{C}_{3} \mathrm{~F}_{8}\right)$ combined with laser photocoagulation in patients with retinoschisis and serous macular detachment due to congenital ODP.

\section{Materials and methods}

This case series was conducted at the Zhongshan Ophthalmic Center of the Sun Yat-sen University in Guangzhou, China and was approved by the Institution Review Board (IRB) of Zhongshan Ophthalmic Center. Prior to the medical procedures, the written informed consents were obtained from the participants or the guardians on behalf of the minors/children enrolled in our study. Nine eyes of eight patients with identified reduction of vision due to ODPM were included in the study. We confirmed ODP based on clinical evidence, including patient symptoms, fundus changes, OCT and fundus fluorescein angiography (FFA). Best-corrected visual acuity (BCVA), OCT and fundus photography were undertaken pre and postoperatively in each patient. Some patients also had visual fields, FFA and multifocal electroretinogram (mfERG) conducted pre and postoperatively.

All surgery was performed at the Zhongshan Ophthalmic Center by the same retinal specialist. Intravitreal gas injection was given under topical anesthesia after obtaining informed consent. Paracentesis and discharge of $0.05-0.08 \mathrm{ml}$ acqueous humor was followed by injection of $0.5 \mathrm{ml}$ of $66 \% \mathrm{C}_{3} \mathrm{~F}_{8}$ gas into the vitreous cavity. After this procedure, the patients were asked to maintain a face-down position for at least $8 \mathrm{~h}$ per day for 2 weeks. Posterior pole retinal photocoagulation was performed 1-2 weeks postoperatively using a $532 \mathrm{~nm}$ wavelength laser (Oculight SL, Iris Medical Instruments, Mountain View, CA, USA). Two to three rows of $100-\mu \mathrm{m}$ spot size burns were placed temporal and adjacent to the optic nerve to span the juxtapapillary border of the retinal detachment and the superior and inferior margins of the detached retina. Exposures of $0.1 \mathrm{~s}$ were used, and the power adjusted to cause Grade I-II gray burn at the level of the retinal pigment epithelium. All the patients were followed up on the 7th and 14th day post-gas tamponade and then fortnightly for a month. Patients were then seen at 1, 3, 6, 12, 18, and 24 months postoperatively. The main outcome measures included BCVA and the status of retinal anatomic features on Stratus OCT (Carl Zeiss Meditec Inc., Dublin, CA, USA), and Spectralis OCT (Heidelberg Engineering, Heidelberg, Germany). Tracking technology with Spectralis OCT was used to assess the progress of maculopathy and parameters, such as foveal and macular thickness, and macular volume were recorded to evaluate the efficacy of the treatment. Patients were given repeated treatment if maculopathy such as serous detachment or schisis still existed or once again became severe.

Data analysis was performed with SPSS statistical software version 16.0 (SPSS Inc., Chicago, IL, USA). Paired- and independent-sample $t$-tests were used to compare group differences. A $P$-value $<0.05$ was considered statistically significant.

\section{Results}

\section{Clinical characteristics}

The clinical characteristics of all eight patients are shown in Table 1, while clinical photographs of representative patients are shown in Figures 1 and 2. The patients were 11-38 years of age (mean, 25.8) and included four females and four males without any family history of ODP. The mean follow-up period in this study was 39.6 months (range 21-62 months). All patients reported decreased VA or metamorphopsia in the affected eye. The duration of defective vision was from 2 days to 21 months. 


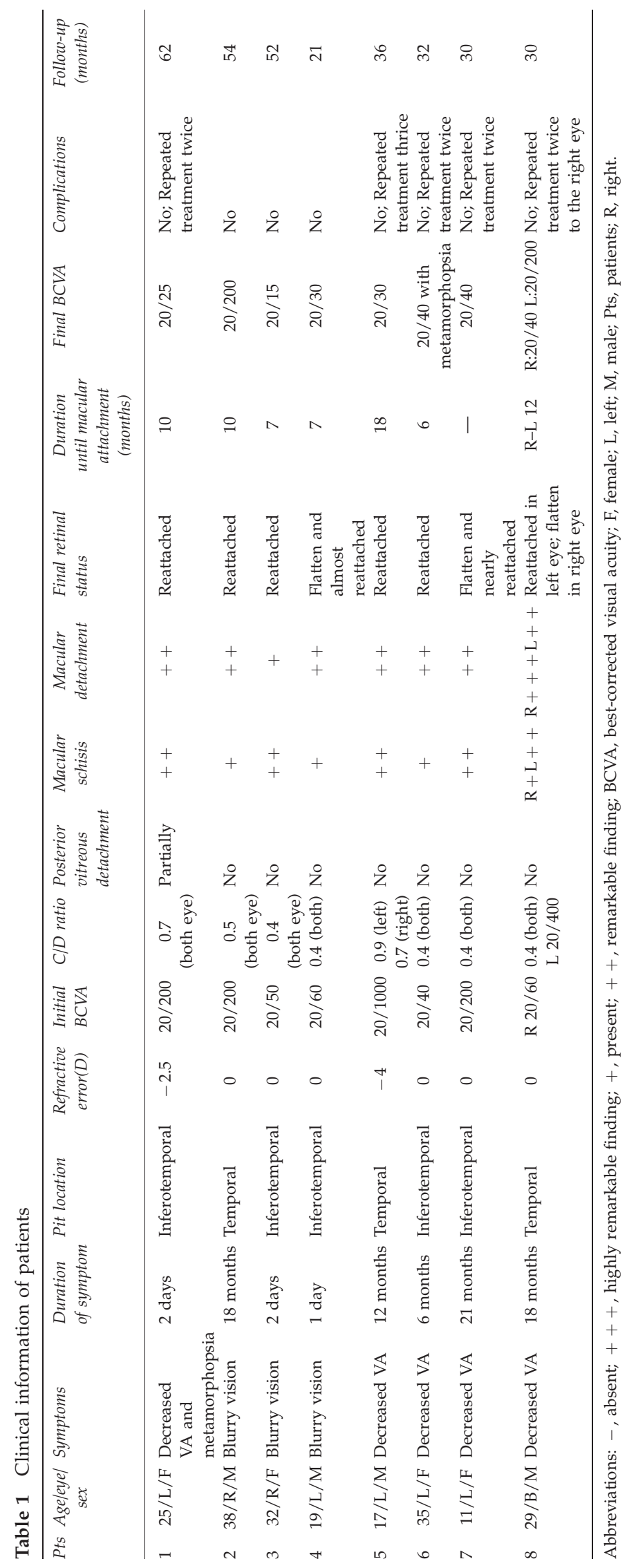




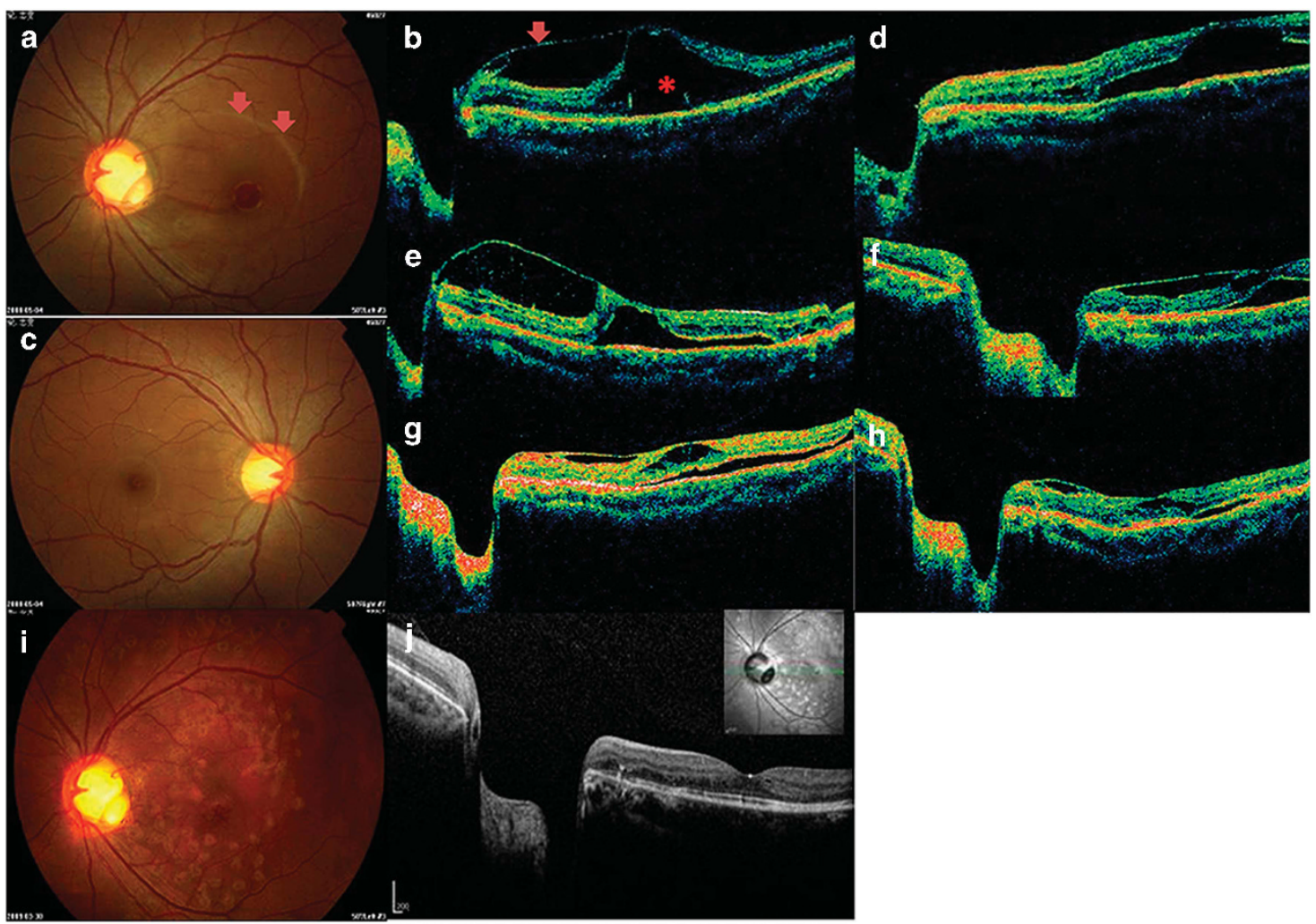

Figure 1 Composite of representative clinical findings from patient 1 . Preoperative fundus findings in the left eye. A $1 / 5$ PD yellowish depression at the infratemporal optic disc with serous retinal detachment involving the macula (arrow) is visible (a). C/D ratio in both eyes (a, c) is about 0.7 . Visual acuity in the affected left eye at presentation was 20/200. Preoperative horizontal OCT in the left eye (b) demonstrated partial vitreomacular traction (arrow) and serous detachment ${ }^{*}$ ) involving the macula. The intra-retinal fluid was most prominent at the level of the outer plexiform layer with some vertical bridging tissue spanning a schisis-like cavity. OCT 10 days postoperatively (d) showed marked reduction of the schisis-like cavity. However, the cavity and serous retinal detachment still existed 1 month postoperatively (e). C3F8 intravitreal injection and photocoagulation was repeated. A significant anatomical improvement was achieved, and the visual acuity gradually improved to 20/40 2 weeks after the 2nd injection (f). Marked absorption of intra-retinal and sub-retinal fluids was noted by OCT 2 months after the repeat injection (g). OCT demonstrated a retinal thickness of $268 \mu \mathrm{m} 4$ months' after repeated treatment (h) versus $744 \mu \mathrm{m}$ (b) before treatment. No recurrence or visual field defects were noted during the follow-up period. Fundus photograph and OCT are shown in i 8 months after repeated injection. The last OCT (Spectralis) at 50 months' follow-up is shown in j.

Patient 1 and 5 had low to moderate myopia, while none of the other patients had any significant refractive error. The cup-to-disc $(\mathrm{C} / \mathrm{D})$ ratios of most patients were normal except in patient 1 (C/D ratio 0.7 in both eyes, Figure 1) and patient 5 (C/D ratio 0.9 in the affected eye and 0.7 in the unaffected eye). A $1 / 2$ PD retinal atrophy inferior to the optic nerve was seen in patient 2 .

Patient 8 had bilateral OPDM and only noted visual disturbance after blunt ocular trauma to the left eye. Examination of the fellow eye of all other patients was unremarkable, with no past history of ocular diseases or treatments.

The presence of a double-layer detachment, consisting of both an inner retinal schisis and an outer layer detachment (neurosensory detachment), was confirmed by OCT either before or after surgery in all nine eyes. Patient 8 had bilateral outer layer detachment with hyperautofluorescent deposits both pre and postoperatively. Preoperative OCT showed no vitreomacular or vitreopapillary traction in all patients except patient 1 .

\section{Anatomic results}

Complete retinal reattachment was achieved in seven out of nine eyes. Among these seven eyes, reduction of the inner layer schisis and outer layer detachment was observed immediately after surgery, with complete fluid absorption after 6-18 months. Among the seven successfully treated eyes, repeat treatment was required in patients 1,5, and 6, which lead to anatomic and BCVA improvements that remained stable during follow-up. The two eyes in which retinal reattachment was not achieved (patients 7 and $8 \mathrm{OD}$ ) showed 


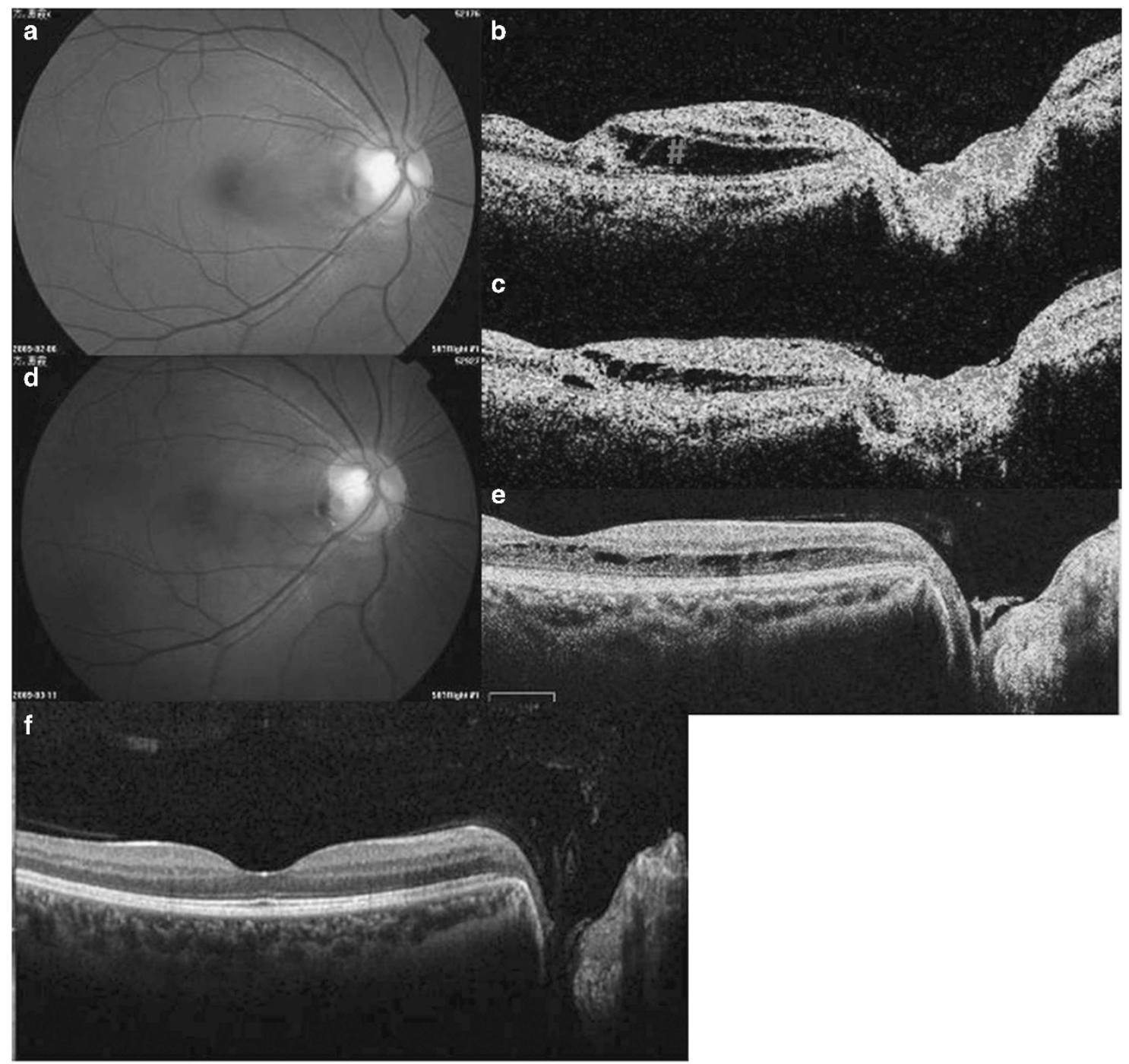

Figure 2 Composite of representative clinical findings from patient 3. A 32-year-old female presented to our clinic with acute onset of blurry vision for $48 \mathrm{~h}$ without metamorphopsia in the right eye. Fundus photography on the first visit is shown in a and with the arrow pointing to an area of maculopathy. The BCVA was 20/30 on the first visit. A tiny optic disc pit at the infratemporal edge of the optic disk is noted with underlying retinoschisis (\#) between the optic head and macula (b) in the right eye. C3F8 tamponade, followed by the face-down position maintenance, was performed. Partial absorption of intra-retinal and sub-retinal fluids was noted by OCT 2 weeks after surgery (c). BCVA in the affected eye improved to 20/20 at that time. Fundus photography (d) and OCT findings (e) showed gradual resolution of retinoschisis 2 months postoperatively. The BCVA further improved to 20/15 and remained stable during the 40-month follow-up period. OCT at the final follow-up is shown in $\mathrm{f}$. There was no reduction in visual field noted after retinal photocoagulation.

a significant decrease in detachment and schisis associated with improved BCVA after repeated treatment, although complete retinal reattachment was not achieved. OCT parameters including foveal thickness, highest thickness, and macular volume change all showed a significant decrease postoperatively (Figure 3). The decrease in foveal thickness pre and postoperatively can be seen in Figure 3a $(P<0.05)$. The treatment also showed significant decrease in areas with the highest thickness of schisis or detachment (Figure 3b, $P<0.05$ ). Macular volume in areas of 1,3 , and $6 \mathrm{~mm}$ was also significantly decreased after treatment (Figures $3 \mathrm{c}-\mathrm{e}, P<0.05$ ).

All patients demonstrated inner segment/outer segment line (IS/OS) restoration with time and the final IS/OS status at the fovea was related to the BCVA. In patient 8 , there was a limited defect in the IS/OS at the fovea in the left eye. Thus, BCVA was slightly increased postoperatively, although the sub-retinal and intraretinal fluid was completely absorbed (Figures $4 a$ and b). All other patients with improved BCVA (Figures 1 and 2) had recovery of the foveal IS/OS after treatment. 
a

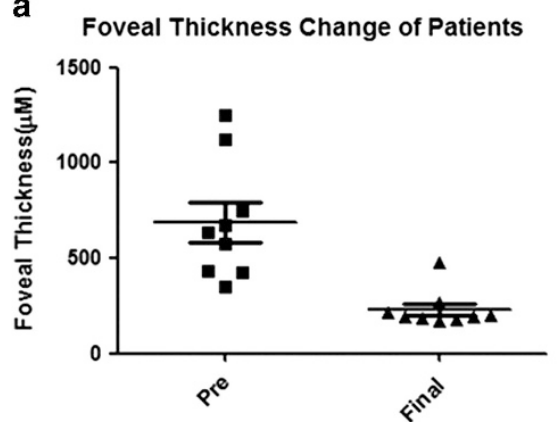

C

$1 \mathrm{~mm}$ Macular Volume Change of Patients

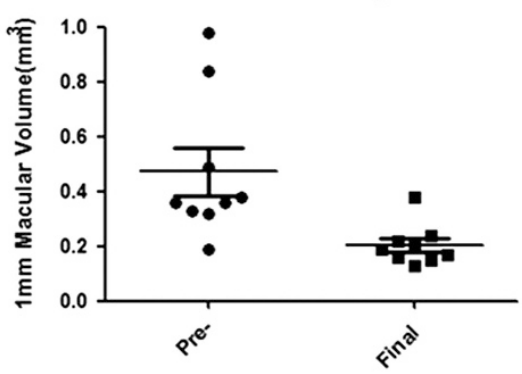

e $6 \mathrm{~mm}$ Macular Volume Change of Patients

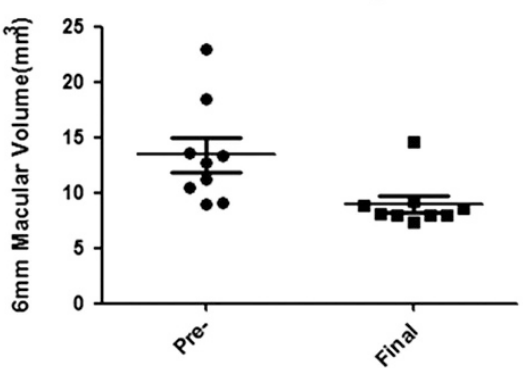

b

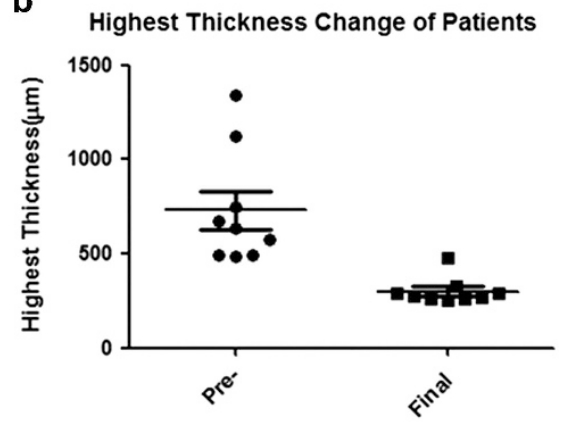

d

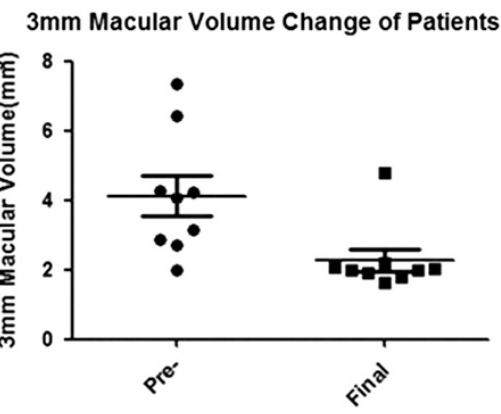

f

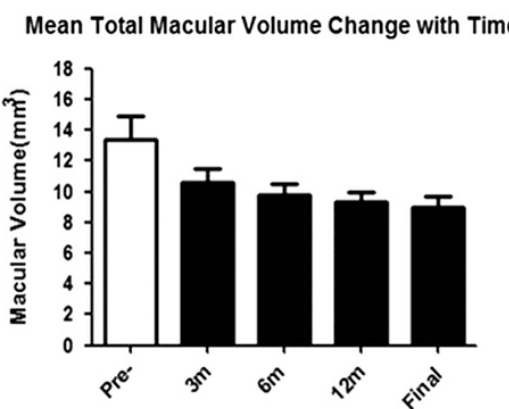

Figure 3 Retinal thickness and macular volume analysis of patients pre and postoperatively. foveal thickness (a) and highest thickness (b) of all patients preoperatively and postoperatively at last follow-up. Macular volume change of $1 \mathrm{~mm}$ (c), $3 \mathrm{~mm}$ (d), and $6 \mathrm{~mm}$ (e) before and after treatment is shown. Mean total macular volume change at different time points (f).

\section{Visual acuity results}

Preoperative and final BCVA are shown in Table 1. The final BCVA improved in seven eyes $(78 \%)$, and remained unchanged in two eyes (22\%). The BCVA started to improve within days (only 5 days in patient 3 ) to months (6-10 months) after treatment despite some residual schisis-like separation and some persistent macular detachment. The BCVA corresponded with the reduced macular elevation and improved IS/OS at the fovea on OCT (Figures $4 a$ and b).

\section{Functional results}

We obtained mfERG results from three patients pre and postoperatively, all of which showed significant improvement (Figures $4 \mathrm{c}$ and $\mathrm{d}$ ). The mfERG electrical response densities were improved in areas 1 (fovea) and 2 (parafovea). Visual field was assessed in five patients with no significant defect or loss of central visual field observed following photocoagulation.

\section{Complications}

Post-treatment complications such as raised intraocular pressure, endophthalmitis, iatrogenic retinal tears, or extended retinal detachment were not observed in the follow-up period. Repeat treatment was required in five eyes. However, the repeat treatments lead to complete reattachment in three eyes and significantly increased $\mathrm{BCVA}$ and resolution of macular elevation in the remaining two eyes. 
a

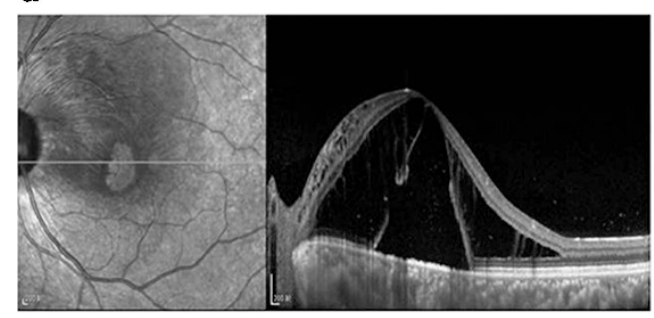

C
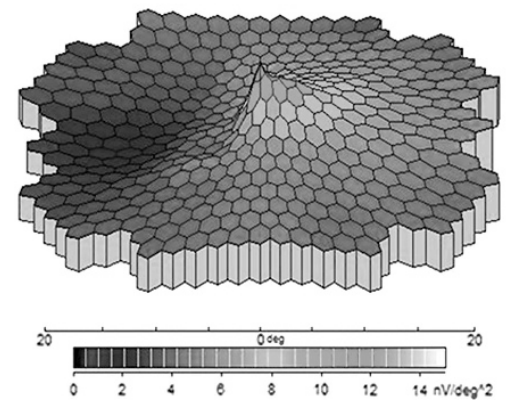

b

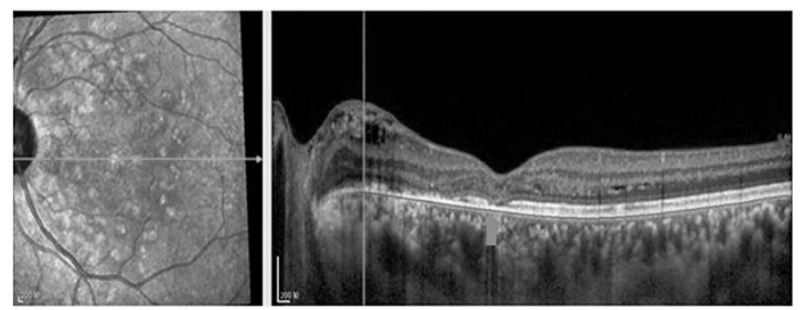

d
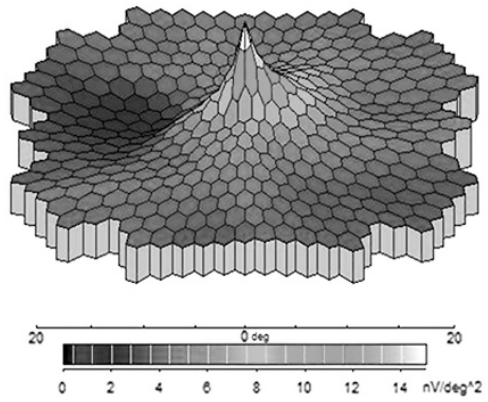

Figure 4 OCT and mfERG of patient 8 . The preoperative (a) and final post-operative (b) OCT of patient 8 (left eye) showed that the IS/OS was not completely recovered (disruption of the IS/OS connection was seen at the fovea (arrow)), which explained the limited VA recovery in spite of complete fluid absorption. mfERG results show that the response improved significantly postoperatively (c) compared with preoperative results (d).

\section{Discussion}

A lack of understanding of the mechanism and treatment of ODPM with retinoschisis and neurosensory detachment pose a significant challenge to clinicians. The role of the vitreous in the pathogenesis of ODPM is important and vitreous abnormalities such as vitreomacular adhesion, partial or complete PVD, and condensed vitreous strands firmly attached to the ODP area have been found in $74 \%$ of the patients with ODPM. ${ }^{2}$ Thus, the release of vitreous traction by vitrectomy, scleral buckling, or macular buckling has been employed widely for the treatment of ODPM. ${ }^{19}$ Vitrectomy combined with gas tamponade, ${ }^{10,13}$ endophotocoagualtion, ${ }^{11}$ silicone oil, ${ }^{20}$ ILM peeling, ${ }^{21}$ or sub-retinal fluid drainage ${ }^{22}$ have also been performed with a relatively high success rate.

Our results show that vitrectomy is not the only way to relieve vitreous traction, especially in cases where no obvious tractional force was noted on OCT. Vitreous traction was found only in some patients with ODPM, ${ }^{23}$ further raising concerns about the use of vitrectomy in all cases of ODP. In our nine eyes, only one patient had vitreomacular traction before treatment (patient 1).

We herein report the successful use of intravitreal gas tamponade without vitrectomy to induce the collapse of retinoshisis and pneumatic displacement of retinal detachment in ODPM. Pneumoretinopexy can facilitate the dissection of the posterior hyaloid, while the facedown position enables the absorption of sub-retinal and intra-retinal fluid, a decrease in the inner layer separation, passive migration of the sub-retinal fluid by channels, and reattachment of the RPE to the sensory retina. Furthermore, the gas can simply function to let the laser work more effectively. In our eight patients, facedown position was maintained for at least $8 \mathrm{~h}$ per day for a minimum of 2 weeks after intraocular gas injection. Macular elevation was flattened in all the patients (as early as 1 week in patient 3 ), while release of vitreomacular traction was noted after only 2 weeks in patient 1 .

After gas absorption, fluid may continue to flow from the remaining inner layer separation to the outer layer detachment. Photocoagulation can block the abnormal communication between the pit and the adjacent inner retinal layers and is thus critical in reducing the inflow of fluid from the pit to the macula. Though the effects of gas injection may be temporary, they have an adjuvant effect with photocoagulation, since the sealing of this subtle fluid channel is critical in the treatment of ODPM. As mentioned previously, regardless of the underlying pathophysiology, sub-retinal fluid appears to gain access to the macula via the anomalous optic pit. Through the adhesion of retina and choroid at the optic disc border, the inflow of fluid from the pit to the macula is reduced. Combination therapy with gas and photocoagulation can also limit the enlargement of the area of detachment and prevent re-detachment of the retina. In our study, photocoagulation at the edge of the ODP was performed 1-2 weeks after gas injection, which is the optimal time window that allows the re-establishment of normal retinal architecture after gas tamponade. 
The natural history of ODP-related macular detachment is not clearly understood. However, persistent neurosensory retinal detachment and/or retinoschisis may cause neural retinal degeneration (with photoreceptor cell loss), retinal pigment epithelial atrophy, or even macular hole formation, which renders a poor visual prognosis. According to recent studies, macular detachment needs to be fixed within 3 days to gain a favorable visual outcome without functional compromise. ${ }^{24}$ In our case series, patients 1 and 3 received prompt treatment at $48 \mathrm{~h}$ after symptom onset, with the achievement of complete visual recovery. In contrast, patients 2,7 , and 8 had prolonged macular detachment for one and a half months prior to receiving treatment, and as a result, had more limited visual improvement even though they demonstrated favorable anatomical rehabilitation after treatment.

Lesion size, macular involvement, and severity of retinoschisis, may also be factors affecting therapeutic response in ODP-related macular detachment. Nevertheless, our experience suggests that early diagnosis and treatment are pivotal to both anatomic and functional recovery. Our OCT findings show that IS/OS layer recovery is another factor determining visual outcome. In patient 5 who was not given prompt treatment (12 months after symptom onset), VA recovery was still adequate because the IS/OS layer of the macula recovered with repeated treatment. In contrast, the left eye of patient 8 had relatively poor IS/OS recovery associated with delayed treatment and a suboptimal BCVA outcome (Figures $4 a$ and $b$ ).

Although repeat treatment was required in five patients, the final BCVA is encouraging and no complications were noted in the long-term follow-up. Meanwhile, the combined therapy is a minimally invasive, simple, and economic method to treat the patients. Thus, it is a worthwhile alternative treatment to ODPM patients considering economic and convenient factor.

\section{Conclusion}

We present encouraging outcomes with minimally invasive surgery in ODPM patients. Although our case series was relatively small, the anatomical and functional results presented in our study demonstrate that gas tamponade with face-down position followed by sequential photocoagulation therapy has the potential to be an initial treatment of ODP. The combination procedures have been shown to be effective and are minimally invasive, providing an alternative to the relatively riskier option of vitrectomy. Despite some patients requiring repeat treatment in our study, the final status of BCVA is encouraging with long-term follow-up.
However, additional studies with larger samples are necessary to further evaluate the effectiveness of our combination therapies as compared with the previously described approaches. Unfortunately, the low incidence of ODPM is a major obstacle for conducting randomized clinical studies of different treatment combinations to firmly establish a treatment of choice for this disease.

\section{Summary}

What was known before

- Several therapeutic approaches have been attempted, but responses have been variable.

- A relatively high incidence of recurrence has been reported after conventional laser photocoagulation or gas tamponade alone.

- Vitrectomy with/without a combination of laser treatment and/or gas tamponade has been reported to be an effective treatment and is widely used.

What this study adds

- Our combined therapy proved to be a minimally invasive, simple, and economic method to treat the ODP patients.

\section{Acknowledgements}

This study was supported by the Fundamental Research Funds of the State Key Laboratory of Ophthalmology, the National Nature Science Foundation in China (Grants No. 81300785; 81170865; 30901642).

\section{Author contributions}

ST and XD designed the study; LL, TL, WM, and XZ were involved in the collection and management of data; LL, TL, YH, ST, and AA analyzed and interpreted the data; $\mathrm{LL}, \mathrm{TL}, \mathrm{XD}$, and AA prepared the manuscript; ST, XD, LL, TL, WM, XZ, YH, and AA reviewed the manuscript.

\section{References}

1 Halbertsma KT. Crater-like hole and coloboma of the disc associated with changes at the macula. Br J Ophthalmol 1927; 11(1): 11-17.

2 Theodossiadis PG, Grigoropoulos VG, Emfietzoglou J, Theodossiadis GP. Vitreous findings in optic disc pit maculopathy based on optical coherence tomography. Graefes Arch Clin Exp Ophthalmol 2007; 245(9): 1311-1318.

3 Bonnet M. Serous macular detachment associated with optic nerve pits. Graefes Arch Clin Exp Ophthalmol 1991; 229(6): 526-532.

4 Sugar HS. Congenital pits in the optic disc and their equivalents (congenital colobomas and colobomalike excavations) associated with submacular fluid. Am J Ophthalmol 1967; 63(2): 298-307. 
5 Vedantham V, Ramasamy K. Spontaneous improvement of serous maculopathy associated with congenital optic disc pit: an OCT study. Eye (Lond) 2005; 19(5): 596-599.

6 Sobol WM, Blodi CF, Folk JC, Weingeist TA. Long-term visual outcome in patients with optic nerve pit and serous retinal detachment of the macula. Ophthalmology 1990; 97(11): 1539-1542.

7 Mustonen E, Varonen T. Congenital pit of the optic nerve head associated with serous detachment of the macula. Acta Ophthalmol (Copenh) 1972; 50(5): 689-698.

8 Theodossiadis G. Treatment of retinal detachment with congenital optic pit by krypton laser photocoagulation. Graefes Arch Clin Exp Ophthalmol 1988; 226(3): 299.

9 Lincoff H, Kreissig I. Optical coherence tomography of pneumatic displacement of optic disc pit maculopathy. Br J Ophthalmol 1998; 82(4): 367-372.

10 Garcia-Arumi J, Guraya BC, Espax AB, Castillo VM, Ramsay LS, Motta RM. Optical coherence tomography in optic pit maculopathy managed with vitrectomy-laser-gas. Graefes Arch Clin Exp Ophthalmol 2004; 242(10): 819-826.

11 Shukla D, Kalliath J, Tandon M, Vijayakumar B. Vitrectomy for optic disk pit with macular schisis and outer retinal dehiscence. Retina 2012; 32(7): 1337-1342.

12 Rizzo S, Belting C, Genovesi-Ebert F, Di Bartolo E, Cresti F, Cinelli L et al. Optic disc pit maculopathy: the value of small-gauge vitrectomy, peeling, laser treatment, and gas tamponade. Eur J Ophthalmol 2012; 22(4): 620-625.

13 Hirakata A, Okada AA, Hida T. Long-term results of vitrectomy without laser treatment for macular detachment associated with an optic disc pit. Ophthalmology 2005; 112(8): 1430-1435.

14 Theodossiadis GP, Ladas ID, Panagiotidis DN, Kollia AC, Voudouri AN, Theodossiadis PG. Fluorescein and indocyanine green angiographic findings in congenital optic disk pit associated with macular detachment. Retina 1999; 19(1): 6-11.

15 Postel EA, Pulido JS, McNamara JA, Johnson MW. The etiology and treatment of macular detachment associated with optic nerve pits and related anomalies. Trans Am Ophthalmol Soc 1998; 96: 73-88.

16 Imamura Y, Zweifel SA, Fujiwara T, Freund KB, Spaide RF. High-resolution optical coherence tomography findings in optic pit maculopathy. Retina 2010; 30(7): 1104-1112.

17 Rosa AA, Primiano Jr HP, Nakashima Y. Pneumatic retinopexy and laser photocoagulation for treatment of optic disc pit detachment: case report. Arq Bras Oftalmol 2006; 69(1): 101-105.

18 Montenegro M, Bonnet M. Optic nerve pits: clinical and therapeutic review of 21 cases. J Fr Ophtalmol 1989; 12(6-7): 411-419.

19 Theodossiadis GP, Theodossiadis PG. The macular buckling technique in the treatment of optic disk pit maculopathy. Semin Ophthalmol 2000; 15(2): 108-115.

20 Johnson TM, Johnson MW. Pathogenic implications of subretinal gas migration through pits and atypical colobomas of the optic nerve. Arch Ophthalmol 2004; 122(12): 1793-1800.

21 Georgalas I, Petrou P, Koutsandrea C, Papaconstadinou D, Ladas I, Gotzaridis E. Optic disc pit maculopathy treated with vitrectomy, internal limiting membrane peeling, and gas tamponade: a report of two cases. Eur J Ophthalmol 2009; 19(2): 324-326.

22 Schaal KB, Wrede J, Dithmar S. Internal drainage in optic pit maculopathy. Br J Ophthalmol 2007; 91(8): 1093.

23 Karacorlu SA, Karacorlu M, Ozdemir H, Burumcek E, Esgin H. Optical coherence tomography in optic pit maculopathy. Int Ophthalmol 2007; 27(5): 293-297.

24 Henrich PB, Priglinger S, Klaessen D, Kono-Kono JO, Maier M, Schötzau A et al. Macula-off retinal detachment-a matter of time? Klin Monbl Augenheilk 2009; 226(4): 289-293. 